\title{
Assessing Pragmatic Interoperability for Process Alignment in Collaborative Working Environment
}

\author{
Shixiong Liu ${ }^{1}$, Weizi $\mathrm{Li}^{1}$, and Kecheng $\mathrm{Liu}^{1,2}$ \\ ${ }^{1}$ Informatics Research Centre, University of Reading, UK \\ ${ }^{2}$ School of Information Management and Engineering, Shanghai University of Finance and \\ Economics, China \\ shixiong.liu@pgr.reading.ac.uk, \\ weizi.li@henley.ac.uk,k.liu@henley.ac.uk
}

\begin{abstract}
This paper is an extension of our previous study on pragmatic interoperability assessment for process alignment. In this study, we conduct four case studies in industrial companies and hospitals in order to gather their viewpoints regarding the concerns when condensing process alignment in a collaborative working environment. Used techniques include interview, observation, and documentation. The collected results firstly are summarised into three layers based on our previous developed pragmatic assessment model, and then are transformed into the elements which constitutes the purposed method, and finally based on the summarised results we purpose a method for assessing pragmatic interoperability for process alignment in collaborative working environment. The method contains two parts: one gives all the elements of pragmatic interoperability that should be concerned when considering process alignment in collaborative working environment, and the other one is a supplementary method for dealing with technical concerns.
\end{abstract}

Keywords: Pragmatic Interoperability, Process Alignment, Collaborative Working Environment, Interoperability Assessment.

\section{Introduction}

Process alignment in collaborative working environment is a complex domain due to human and technical factors and multi-stakeholder involved, and multidisciplinary nature of the problems. Organisation is such a complex area where business processes and functions require multi-systems collaborations, and highly relies on effective information communications among departments, and therefore faces many challenges regarding pragmatic interoperability such as information collision, policy obstacles, and procedure mismanagement. Our previous study has developed the semiotic interoperability framework [1][2] and several methods for measuring interoperability at different organisational levels [3][4]. This paper is an extension of our previous study on pragmatic interoperability assessment for process alignment. In this study, we conduct four case studies in industrial companies and hospitals in order to gather their viewpoints regarding the concerns when condensing process alignment in a collaborative working environment. Used 
techniques include interview, observation, and documentation. The collected results firstly are summarised into three layers based on our previous developed pragmatic assessment model, and then are transformed into the elements which constitutes the purposed method, and finally based on the summarised results we purpose a method for assessing pragmatic interoperability for process alignment in collaborative working environment. The method contains two parts: one gives all the elements of pragmatic interoperability that should be concerned when considering process alignment in collaborative working environment, and the other one is a supplementary method for dealing with technical concerns.

\section{Background}

In the study of the interoperability, most of the work focuses on discussion at a technical level. Although some of them have extended to deal with semantics, a very limited number of publications elaborate the interoperability at the pragmatic level [2]. Undoubtedly the research on technical and semantic interoperability can help establish a better understanding of data exchange and data interpretation, as well as leading to the development of supporting technologies and standards. However, the collaboration of different working processes require an assessment of pragmatic interoperability that ensures supported process can act upon the semantic information in order to deal with the complexity. Before defining pragmatic interoperability, our previous work has purposed the concept of semiotic interoperability [1], and has applied the concept in healthcare domain for analysing interoperability of collaboration at radiology department [4]. The concept of semiotic interoperability is based on the semiotic framework [5]-[7] that explains all aspects of how signs can be used and communicated for successful communication. Using the concept allows different parties/processes/organisations to work together through communication with insight into six levels: physical, empirical, syntactical, semantic, pragmatic, and social. At the pragmatic level, the interoperability is concerned with the relationship between signs and the potential behaviour and intention of responsible processes. Thus we defined the pragmatic interoperability as a level concerning the aggregation and optimisation of various business processes for achieving intended purposes of different information systems. In addition to our definition of pragmatic interoperability, other researchers have also contributed. Benson [8] defines it as coordination of work processes across different people to enabling work collaboration. Sadeghi et al. [9] state the pragmatic interoperability in healthcare is the ability among healthcare processes and various actors (i.e. healthcare providers and patients) that interact with information systems. Successful communication at this level is achieved if the hearer understands the speaker's intentions, which goes beyond the semantic interpretation of the communicative act. Interoperability is achieved at this level when processes serving different purposes under different contexts by different information systems can be composed to jointly support a common intention. 


\section{Methods}

The study is mainly conducted through interviews and surveys in two industrial companies and two hospitals in China. One of the companies is an automobile manufacturer, and the other one is a software company that provides integration solutions for various building control systems. The background of the automobile manufacturer is that they have enabled automated information sharing among three parties: dealership repair facility, automobile rental companies, and themselves. The background of the software company is that they are currently running several integration projects which manly aim to enable information sharing among systems such as Building Management System (BMS), Energy Monitoring System (EMS), Invasion Surveillance System (ISS), etc. The background of the hospitals is that they tend to achieve information sharing among Radiology Information Systems (RIS), Electronic Health Record (EHR), and Picture Archiving and Communication Systems (PACS). The Radiology department provides diagnostic and interventional radiology for inpatients, outpatients and general practitioner referrals. Various healthcare services such as Computed Radiography (CR), Computed Tomography (CT), X-ray, and Interventional Radiology produce a huge amount of information regarding patient's healthcare delivery and clinical process.

This study adopts a qualitative research approach including various techniques [10]. During the data collection process, we reviewed the most up-to-date working documents published by the companies and hospitals, conducted an observation, and interviewed 31 experts in order to gather their viewpoints as inputs for developing the assessment method. Details of each technique are presented in following:

Documentation \& Literature: The documentation included literature review covering research publications, official reports, and working papers from the companies and hospitals. The research publications were sourced from several electronic databases in terms of information systems, process integration/alignment, and health informatics. The working papers were provided by the companies and hospital IT services department.

Observations: We conducted one participant observation in working on several projects for the software company; and other three non-participant observations in the automobile manufacturer and hospitals focusing on their work processes, staff routine, individual activities, and technologies/techniques used for information sharing.

Interviews: 31 semi-structured interviews (summarised in table 1) were conducted with relevant stakeholders. The interviews lasted around 40 minutes and were on a one-to-one basis. The interviews were tape-recorded and later transcribed and rendered anonymously. The results of the interviews are summarised into categories which are used as key inputs afterwards for developing the pragmatics assessment framework.

After summarising all the key elements from interviews, this study also conducted surveys to all the interviewees asking them to scale how much each element would affect the process alignment based on their experiences. However, the results of the survey will be presented in future work. 
Table 1. Summary of interviews

\begin{tabular}{lllllll}
\hline Sectors & Sample & \multicolumn{5}{c}{ Years of experience } \\
\hline & & $0-5$ & $6-10$ & $11-15$ & $16-20$ & $20+$ \\
Industry & 22 & 7 & 11 & 2 & 1 & 1 \\
Hospitals & 9 & 3 & 2 & 1 & 1 & 2 \\
Total & $\mathbf{3 1}$ & $\mathbf{1 0}$ & $\mathbf{1 3}$ & $\mathbf{3}$ & $\mathbf{2}$ & $\mathbf{3}$ \\
\hline
\end{tabular}

\section{$4 \quad$ Findings}

As mentioned previously, this section presents the results from interviews, observations as well as literature reviews. The key concerns and barriers when considering working collaboratively in organisations are identified, and then each of the identified concerns are pinpointed into the Pragmatic Interoperability Measurement Model developed previously [4] as the key elements for assessment. This section firstly recaps the three layers of organisation (Informal, Formal, and Technical) which is used for setting the outline of all identified key concerns, and secondly these concerns are categorised more specifically into each element for developing assessment method based on the process of achieving intended purposes, at last some of the key elements are selected for discussion.

Our previous work purposed the pragmatic interoperability assessment model [2] based on Organisational Onion [6] defining three layers for understanding and capturing key concerns when considering process alignment and working collaboration. As depicted in Figure 1, informal layer contains aspects such as community, social norm, policy, and culture. Those aspects can be expanded to be different behaviour patterns of both organisations and individuals. The pragmatic interoperability is to align the different aspects in order to solve conflicts of cohesiveness. In formal layer, business functions and procedures play dominant role that specifies on how functions should be carried out and how tasks should be performed. The pragmatic interoperability is to align procedures and rules in order to achieve higher efficiency. It defines business goals, model business processes and brings the collaboration of administrations that aims to exchange information and have different internal structures and processes. Besides, this layer also supports the upper layer by addressing the requirements of the user community such as making services available, accessible, identifiable and useroriented. The technical layer mostly refers to the technical computer systems and their technical functions. The systems and functions can be programmed according to norms and procedures. The pragmatic interoperability does not directly refer to this layer but undoubtedly requires continuous support from it which is to align technical functions and business processes in order to achieve higher system productivity. It supports seamless sharing of data, which is automated sharing of data between information systems based on a common exchange model. It also covers the technical issues of linking computer systems and services. A few key aspects are included such as interconnection services, data integration, open interface, data presentation and exchange, and accessibility will be dealt within this level.

Based on the results from interviews, the following summaries a list of key concerns of pragmatic interoperability when considering processes alignment and working collaboration. They are split into three levels as discussed previously. 


\begin{tabular}{|l|l|}
\hline Informal & Community, social norm, policy, culture \\
\hline Formal & $\begin{array}{l}\text { Organisational strategy/vision, business } \\
\text { governance, domain analysis, organisational roles, } \\
\text { functional profile, rules, procedures, }\end{array}$ \\
\hline Technical & $\begin{array}{l}\text { Data semantics, information infrastructure, } \\
\text { information model, schema, script, interface, } \\
\text { platform, deployment model, }\end{array}$ \\
\hline
\end{tabular}

Fig. 1. Pragmatic Assessment Model [3]

\section{- Informal}

To enable collaboration between different processes/systems/organisations, a shared intended purpose plays a key role that supports perceiving of personal beliefs and organisational ground rules, whereas an un-shared purpose may be considerable conflicts between the organisational level and personal level. Issues like restriction to staff behaviour, information collaboration (information channels alignment), varieties of purchased information systems (different venders and services providers), and privacy and security concerns should be solved in this level. Taking one example from one of the interviewed hospital, the informal level is to concern with the understanding of the healthcare, regulatory, legislative and healthcare environment in which information systems need to be deployed to support healthcare delivery. It requires agreement on key organisational concepts such as policies, processes and roles; it also captures relevant patterns such as compliance, governance, legislative and change management. There are other concerns summarised in table 2.

Table 2. Other concerns at informal level summarised from interview results

\begin{tabular}{ll}
\hline Concern & Explanation \\
\hline Culture issue & Tacit knowledge has not been explicitly stated and shared \\
Ethical issue & Appropriateness of taking actions on healthcare service delivery \\
$\begin{array}{l}\text { Behavioural factor } \\
\text { Management style }\end{array}$ & Willingness to be open and to share \\
& Leadership style influencing the degree of willingness of collabora- \\
tion & Internal control process, work flow, staff relationships, communica- \\
Policy and procedure & tion patterns, cut-across political boundaries, etc. \\
$\begin{array}{l}\text { Restriction to staff } \\
\text { viour }\end{array}$ & $\begin{array}{l}\text { beha-Staff's fear on integrated working process as restriction that might } \\
\text { Privacy and security }\end{array}$ \\
\hline
\end{tabular}

Policy is one the most important and most widely mentioned key concerns in discussion of interoperability at informal level. From its perspective, collaboration always faces obstacles, because it cuts across political boundaries, and causes amount of changes (e.g. internal control process, work flow, staff relationships, communication patterns) in organisations [11]. For instance, in the healthcare, before the integration of RIS, the political issues between clinicians and radiologists have been raised for long due to the autonomous role of clinician. Comparing with the radiologists, clinicians have the right to choose and take responsibility for their treatments made, and they have direct access to 
the policy making. The integration of RIS will enable the information sharing between the clinicians and the radiologists, so that patient's information and treatments made by clinicians will be transparent for the radiologists. However, it is challenged as clinicians have concerns that the information they collect and the treatments they made are proprietary and thus unavailable for inclusion in the process of information sharing. The integration of RIS also solves a political issue that clinicians who are geographically separated from the hospital were having conflicts for decision making. A quote from one clinician is: "... All decisions have to be made with support of our staff... the integration has a chance to work here only if the staff can see the benefits..."

In line with the policy concern, another one comes from the medical staff is that they are seeing the collaboration of working processes as restriction that would control their behaviour. They are more interested in research than administration, and they await more benefits to patient care delivery from the information systems integration, rather than the improvements of communication with the staff. This is the decisive factor that will let them accept the collaboration. Besides there are also concerns regarding culture, privacy, and security, but there are not as highlighted as the previous concerns, thus we will elaborate them in this paper.

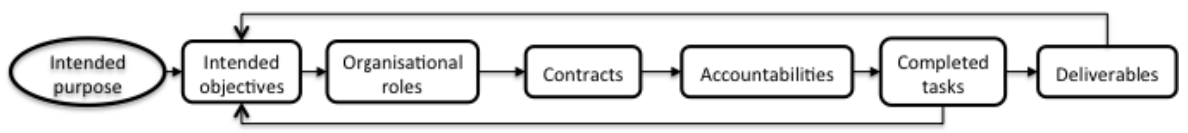

Fig. 2. Summarised procedure for meeting intended purpose from interview

\section{- Formal}

As discussed previously, to meeting shared purposes at formal level, organisations have a more specific procedure to follow. The procedure begins with setting up several intended objectives for the shared intended purpose, then allocates these objectives to relevant organisation roles, and signs contracts which define explicit tasks. The tasks relate to accountabilities for the completeness, quality, integrity and security of information that originates in one party and is transmitted to and used by another. Finally, the completed tasks with their deliverables are evaluated by matching the original defined intended objectives.

Besides the elements of this procedure, there are other concerns captured from the result of interviews (table 3 ).

Table 3. Other concerns at formal level summarised from interview results

\begin{tabular}{ll}
\hline Concern & Explanation \\
\hline Organisation structure & Centralised, decentralised, hierarchical, matrix, networked, etc. \\
Harmonized strategy & Aligned operations to be applicable on the strategic level \\
Performance constraints & Fewer investment but more effective collaboration \\
Cost constraints & Unexpected budget \\
$\begin{array}{l}\text { Data source interoperability } \\
\text { Context awareness }\end{array}$ & Multiple data sources used for supporting process \\
Varieties of purchased systemsPurchased systems from various venders with low capability \\
\hline
\end{tabular}


To assess pragmatic interoperability, we should not only concern the information exchanged between technical systems, but also the knowledge of the context that the information exists within for each system or process. As quoted from one IT project managers, "...It is important to articulate the requirement for context awareness that process representation begins. The context of the target system should also be made available to the origin system. Key questions such as what process will first operate on the information at the target system once it receives it, and what state of preceded processes are should be concerned..." By understanding this context, the system engineer and integration designer can ensure pragmatic interoperability is addressed for the needs of process integration. To define the context, one manager of the logistics department said that "Assume that the context is about internal workings of the process, in other words, the initialization state, the end state, the nature of data transformations, and details about the timing of the process are all considered, so that the receiving process can make better use of information it receives. This information is in context, but it also shows the dynamic nature of that context to the receiving system, because it now has specific information about the dynamic context within the originating system." For example, context is seen as a demand for more information between the Model Manufacturing Demand Service and the Rental Fulfillment Service, so that a specific understanding of the models requested has a deeper meaning. This could be a specific based on the timing of the data, the initialization state of the Rental Fulfillment Service, and the data transformations. This gives a more dynamic picture of the context for the information being produced by one process for another, and allows for a deeper understanding of the meaning of that context.

Another example in the healthcare environment explains the concern of different data sources. The patient-centred care delivery is tailored to patient and requires collaboration of several information systems, and other involved team members also require the data of patient such as status and treatment. To deliver care for an individual patient, various activities need to be collaborative and to keep updating the database in real time. However, this collaboration is challenged duo to the complexity, it requires multiple data sources integrated to support the different requirements. "... Originally, our IT environment operated with costly point-to-point interfaces, and we also lacked control of troubleshooting while the messages transmission failed... we operated within a manual environment where we needed to manually enter orders with their images received from outside reading facilities into our system, and had to fax copies back to the outside facilities..." quoted from one clinician in radiology department. Information communication is also challenged due to the varieties of purchased systems for hospital. Those systems were provided by several small venders, who remained their competitiveness by selling only one type of systems or focusing on one specific function. This results in that the stakeholders get frustrated more often because the communication of patient's data/information failed among various systems. As one clinician stated: "We became accustomed to telling our patients that we cannot provide the information for them immediately because their information cannot be accessed, although we have those information in the database... I normally have to download the hard copy and then manually re-input the data for my patient." Furthermore, information communication is also challenged because the clinicians 
and the radiologist were located in different rooms, and therefore the collaboration takes place asynchronously at most of the time, which can be solved via management channel.

\section{- Technical}

The purpose of technical level is to make exchanged data available for supporting the processes at upper level. For example, in healthcare environment, it is concerned with the understanding of technical functionality for supporting information systems. It requires agreement on a core set of technical concepts, such as technical components and devices, the interactions between components, interface and technical services; it also captures relevant patterns such as technical architecture styles and styles of component interactions. However, the study at this level is not the focus of the assessment of pragmatic interoperability, although it is vital to support the understanding the concerns of pragmatic interoperability at upper levels. Therefore, during the interview, only few concerns regarding technical level are summarised below, but we still purpose a method for dealing with those concerns in the next chapter.

Table 4. Summarised concerns at technical level from interview results

\begin{tabular}{ll}
\hline Concern & Explanation \\
\hline Semantic heterogeneity & $\begin{array}{l}\text { Refers to the variation of semantic meaning in information } \\
\text { resources which will lead to the semantic conflicts and compli- } \\
\text { cation for data integration } \\
\text { Approaches that employ ontologies for information systems }\end{array}$ \\
$\begin{array}{l}\text { Ontology structure } \\
\text { Operation/system language }\end{array}$ & $\begin{array}{l}\text { The language using in both systems } \\
\text { Ambiguous terminology }\end{array}$ \\
\hline
\end{tabular}

Next chapter will purpose a method for assessing pragmatic interoperability based on the results collected from this chapter. The method contains two parts: one gives all the elements of pragmatic interoperability that should be concerned when considering process alignment and working collaboration, and the other one is a supplementary method for dealing with technical concerns.

\section{$5 \quad$ A Method for Assessing Pragmatic Interoperability}

The nature of information communication relies on successful signs communication [1], which must start with understanding and modelling the organisation where information communication exists [6]. The organisation onion [7] stresses the distinctions as well as the interdependent links between the business process and IT systems. The organisation morphology provides a useful modelling method for understandings the norm structure of organisation. Each organisation can be characterised as a structure of norms that allow functions to be coordinated for certain purposes [6], and pragmatic interoperability, is to enable the purposes of each process can be understood and perceived during collaboration. Based on the results collected from the interviews and observations presented in previous chapter, we purpose a method for assessing pragmatic interoperability which contains two parts: one gives all the elements of 
pragmatic interoperability that should be concerned when considering process alignment and working collaboration, and the other one is a supplementary method for dealing with technical concerns.

Figure 3 depicts the method purposed for assessing pragmatic interoperability. In the informal level, two key elements are defined: community participation refers to what parties in what roles are eligible to participate and what are the prerequisites for their participation, and policies refer to those policies within each party's jurisdiction that influence the interoperability of the organisation. Organisation may encode business rules that are not explicitly specified but cause incompatibilities in exchanged information. Aligning policies across jurisdictional boundaries is one of the most difficult tasks of collaboration.

In formal level, meeting shared intended purposes is the goal of achieving pragmatic interoperability when two process/organisations working collaboratively. The procedure has been identified based on the interview results. It begins with setting up several intended objectives for the shared intended purpose, then allocates these objectives to relevant organisation roles, and signs contracts which define explicit tasks. The tasks relate to accountabilities for the completeness, quality, integrity and security of information that originates in one party and is transmitted to and used by another. Finally, the completed tasks with their deliverables are evaluated by matching the original defined intended objectives.

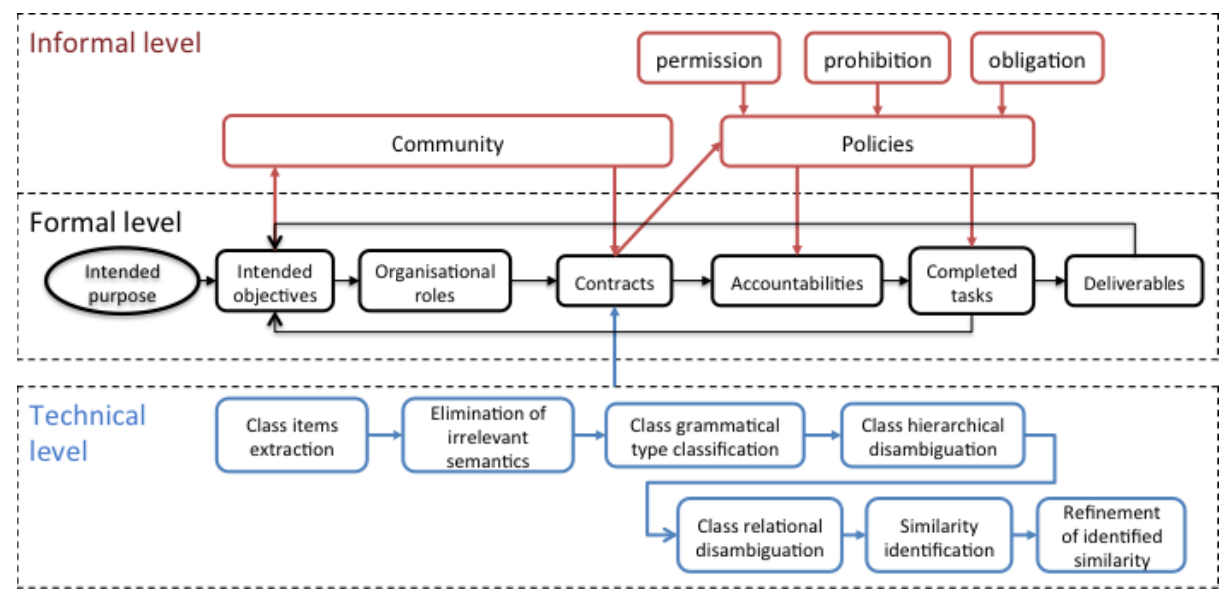

Fig. 3. Method for Assessing Pragmatic Interoperability

In technical level, we purpose a matching process that deal with the concerns summarised from the interview results such as semantic heterogeneity, ontology structure, and ambiguous terminology. The process contains seven steps (class items extraction, elimination of irrelevant semantics, class grammatical type classification, class hierarchical disambiguation, class relational disambiguation, similarity identification, and the refinement). In the class items extraction phase, the goal is to represent all the classes of the ontology in the same form. It is to represent each class like a set of terms. The characters are executed without meaning as so punctuation marks or spe- 
cial characters. When dealing with issues like too many corresponding senses for each class term, the steps of grammatical type classification, hierarchical disambiguation, and relational disambiguation are used for articulating semantics of the information. After semantic disambiguation, different ontologies are compared by using defined metrics in order to identify and refine the similarities. As the matching process using at this level is not the focus of this paper, thus the elaboration of the process itself as well as its application and validation will be presented in future work.

\section{Conclusion and Future Work}

This paper is the extension of our previous research. It proposed a method for assessing pragmatic interoperability for process alignment in collaborative working environment. The study is conducted qualitatively by using techniques of interview, documentation, and observation. The results collected firstly summarised into three layers based on our previous developed pragmatic assessment model, and then categorised them into elements which constitutes the purposed method. The method contains two parts: one gives all the elements of pragmatic interoperability that should be concerned when considering process alignment in collaborative working environment, and the other one is a supplementary method for dealing with technical concerns. Validation and application for the purposed method will be presented in future work.

\section{References}

1. Li, W., Liu, K., Liu, S.: Semiotic interoperability - a critical step towards systems integration. In: The 5th International Conference on Knowledge Management and Information Sharing, pp. 508-513 (2013)

2. Liu, S., Li, W., Liu, K., Han, J.: Evaluation frameworks for information systems integration: from a semiotic lens. In: The 3rd International Conference on Logistics, Informatics and Service Science, pp. 559-568 (2013)

3. Liu, S., Li, W., Liu, K.: Assessing Pragmatic Interoperability of Information Systems from a Semiotic Perspective. In: 15th International Conference on Informatics and Semiotics in Organisation, ICISO (2014)

4. Liu, S., Li, W., Liu, K.: Pragmatic Oriented Data Interoperability for Smart Healthcare Information Systems. In: The 14th IEEE/ACM International Symposium on Cluster, Cloud and Grid Computing (2014)

5. Filipe, J.B.L.: Normative Organisational Modelling using Intelligent Multi-Agent Systems. Staffordshire University: Ph.D Thesis (2000)

6. Liu, K.: Semiotics in Information Systems Engineering. Cambridge University Press, Cambridge (2000)

7. Stamper, R.: Information in business and administrative systems. Batsford, London (1973)

8. Benson, T.: Principles of health interoperability HL7 and SNOMED. Springer (2009)

9. Sadeghi, P., Benyoucef, M., Kuziemsky, C.E.: A mashup based framework for multi level healthcare interoperability. Inf. Syst. Front. 14(1), 57-72 (2011)

10. Yin, R.: Case study research: Design and methods. Sage, pp. 85-96 (2003)

11. Kim, K., Michelman, J.: An examination of factors for the strategic use of information systems in the healthcare industry. MIS Q., 201-216 (June 1990) 